yield by the application of emulsions of various growth-regulating substances, are also being studied. An unusual, if somewhat contemporary, flavour is introduced by the inclusion in the report of instances of sabotage in the experimental fields by terrorists.

\section{VERY-HIGH-FREQUENCY BROADCASTING IN GREAT BRITAIN}

$I^{N}$

its first report last year the Television Advisory Committee made recommendations to the Postmaster-General regarding the future of television broadcasting in Great Britain (see Nature, August 22,1953, p. 337). At that time it was stated that the Committee's next task would be to investigate the introduction of high-frequency sound broadcasting as an additional system to the medium- and long-wave transmissions already used by the B.B.C. for its programmes, and now the Committee has published, as its second report*, its recommendations on this score.

The problem before the Committee and its Tech. nical Sub-Committee is that the existing mediumand long-wave services are barely adequate for supplying the general and regional programmes, that they allow no scope for expansion and that, in fact, they are threatened with progressive deterioration owing to the increased interference from Continental stations, mainly those not bound by the Copenhagen Broadcasting Agreement. It is for the most part accepted that the general plan for tackling this problem is to utilize Band II of the frequency spectrum, namely, the very-high-frequency band of 88-100 Mc./s. $(3 \cdot 4-3 \mathrm{~m}$.) that has been allocated by international agreement to Great Britain for sound broadcasting; because the top part of this band is already used by Civil and other services, this in practice reduces the band to the range 88-95 Mc./s. For most broadcasting, such as the existing B.B.C. programmes (including the sound channel of television), the audio-frequencies are impressed on the carrier wave by the method of amplitude modulation; but very-high-frequency waves do offer the practical alternative of frequency modulation, and the Technical Sub-Committee has gone to great lengths to decide which form of modulation to use. This is a problem of many facets, but the main feature that one must assume is that most receivers will have comparatively poor aerials and that most reception areas will be partly screened and subject to a not inconsiderable amount of electrical interference (for the most part amplitude-modulated). For a service comparable with the sound programmes received on long- and medium-waves, the median value of an amplitude-modulated signal at $90 \mathrm{Mc}$./s. would need to be $1.5 \mathrm{mV} . / \mathrm{m}$. generally and $5 \mathrm{mV} . / \mathrm{m}$. in large towns ; for the same grade of service on frequencymodulation, the signals need only be 0.25 and $0.8 \mathrm{mV} . / \mathrm{m}$., respectively. An interesting point noted in the report is that the signal-to-noise ratio of the sound received in conjunction with television is extremely poor, the amplitude-modulated signals at $45 \mathrm{Mc}$. $\mathrm{s}$. (at which frequency the standard dipole is twice as efficient as one designed for $90 \mathrm{Mc}$./s.) being about 0.1 and $0.35 \mathrm{mV} . / \mathrm{m}$. for the two types of

* General Post Office: Second Report of the Television Advisory Committee (1952). Pp. 25. (London: H.M.S.O., 1954.) 1s. net. locality; but from a psychological point of view, the associated visual picture masks the poor signalstrength of the sound.

There are, in fact, three possible types of veryhigh-frequency broadcasting : frequency-modulation at $+75 \mathrm{kc} . / \mathrm{s}$. deviation, requiring a channel separa. tion of $200 \mathrm{kc} . / \mathrm{s}$.; narrow-band amplitude-modulation (channel separation $15 \mathrm{kc} . / \mathrm{s}$. ) ; and wide-band amplitude modulation (channel separation $200 \mathrm{kc} . / \mathrm{s}$.). For each of these systems to cover 97 per cent of the population of the United Kingdom with three national programmes, the frequency-modulation needs to transmit a total of $760 \mathrm{~kW}$. from 77 transmitters (theoretically 25 per programme, but two extra are needed for Welsh programmes), while the two amplitude-modulation systems require 2,800 $\mathrm{kW}$. from 108 transmitters; the corresponding capital outlays are estimated at $£ 3 \cdot 5$ million and $£ 9$ million, respectively, and the annual costs at $£ 800,000$ and $£ 2$ million. These figures assume a common mast and aerial system for the radiation of three programmes in each area. But the figures for amplitudemodulation are worsened even further if the transmissions in any one area were restricted to a single $600-\mathrm{kc} . / \mathrm{s}$. band ; for then, due to filtering difficulties, it would be necessary to use three separate, widely spaced masts, or increase the power considerably in a single mast, and in either case the capital and annual costs would be very much increased for such an installation, perhaps as much as doubled.

The report devotes considerable space to the question of receivers, because obviously the introduction of very high frequencies would entail the production of entirely new sets or the adaptation of existing ones, and this is a matter of very great financial importance both to the radio industry and to the public. Though narrow-band amplitude modulation appears on the surface to offer certain advantages, there are in fact considerable difficulties, the principal ones being that oscillator drift in the receiver must be held to the fine limits of $2 \mathrm{kc} . / \mathrm{s}$., and it would be very difficult to allocate frequencies which would entail freedom from certain types of interference and also the propagation of interference in the 41-68 Mc./s. band from the receiver oscillator. Thus the choice really narrows down to wide-band amplitudemodulation or frequency-modulation, and as far as receivers are concerned, whether they be adaptors for existing sets or new sets designed for very-highfrequency reception, there seems little to choose between the two systems. As a matter of interest, although adaptors are discussed fully in the report, it is considered that they will be required for only a limited time and that the market for equipment receiving very-high-frequency radiation will eventually be concerned solely with sets designed exclusively for the new type of radiation. Thus the advantage of frequency-modulation is more or less based on the transmitter point of view, though this is not inconsiderable.

The Committee therefore recommends that very. high-frequency broadcasting using frequency modulation should be established in Great Britain, and that this holds good whether sufficient stations are eventually put up to give a national coverage on three programmes or whether the number is sufficient only to provide a substantial reinforcement of the services now given on the long-and medium-waves. This recommendation is signed by nine of the ten members of the Committee, and the dissenting member, Mr. C. O. Stanley, one of the representatives 
of the Radio Industry Council, has submitted a minority report which is printed in full after the majority report, together with a point-by-point reply by the other members of the Committee to Mr. Stanley's views.

The main feature of Mr. Stanley's arguments is that he does not accept the assumption that veryhigh-frequency broadcasting need necessarily be introduced in the first place, and that, if it were, the experience of other countries that have introduced it, most of them using frequency-modulation, is decidedly unfavourable. If the plans for very high frequencies go ahead, then the general public will have to start considering buying a completely new receiver, the cost of which for an amplitude-modulator set would $\mathrm{be}$, on the average, $£ 20$ and for an equivalent frequency-modulation one £30. Mr. Stanley thinks that the future for the existing broadcasting systems is not necessarily hopeless and that the Committee's first task should be to investigate what may be done to improve them rather than replace them. The reply of the majority of the Committee is that veryhigh-frequency broadcesting is an unwelcome but inescapable complication, and that its introduction will probably be spread over at least ten years. During that time existing sets owned by the public will tend to wear out and need replacement, and the higher cost of $a_{b}$ frequency-modulation receiver is more than outweighed by the advantages of the system.

As in the case of the report on television, the Committee's terms of reference are for the most part concerned with technical problems; but the expansion of television and the introduction of very-highfrequency broadcasting both involve heavy capital outlay by the Government and the development by industrý of a wide range of new products, together with the necessity of the general public to find the money for purchasing these products. These are grave economic factors and will, no doubt, be an important, if not over-riding, consideration in any Government decision on these matters.

\section{HORIZONTAL DISPOSITION OF RHIZOMES}

$\mathrm{T}$ THE diageotropic disposition of the rhizome in bishop's weed (Aegopodium podograria) has been attributed by T. A. Bennet-Clark and N. G. Ball (J.Exp. Bot., 2, 169; 1951) to the action of two hormones working in opposition to one another; both are affected by gravity, and the horizontal position of the rhizome is due to an equilibrium between the effects of the two hormones.

N. G. Ball has now taken the investigation of the mechanism of this intriguing problem-the horizontal disposition of rhizomes - a stage further by examining the effects of several known growth-regulating substances on Aegopodium rhizomes (J. Exp. Bot., 4, $349 ; 1953)$. The experimental treatments, which involved the immersing of rhizomes for periods of forty-five minutes in solutions of the growthregulating substances, were carried out in the absence of visible light, the rhizomes being photographed every hour by infra-red radiation. After immersion in $10^{-4} M \beta$-indoleacetic acid the rate of rhizome growth is approximately doubled; but about five hours later it declines to one-third or less of its original value and then gradually recovers.
'This depression in the growth-rate can, however, be reversed by a second auxin treatment, the inference being that the exhaustion of some substance necessary for growth is not involved. It is suggested that the auxin which first accelerates elongation may later be converted into, or may cause the production of, an inhibitor. Thus, when successive immersions in auxin are repeated every two hours, the inhibition can be partially overcome as long as the treatment is continued. As the immersion of horizontal rhizomes in the growth-regulating substance also causes them to turn up, this is taken as giving support to the hypothesis indicated above; that is, an equilibrium is disturbed.

Thus it would appear that when a rhizome is immersed in a solution of auxin there is some mechanism which causes the auxin to accumulate on the lower side. As this may also be assumed to happen to any auxin produced in the rhizome itself, a probable explanation appears to be that the horizontal position assumed by the rhizome results from the balancing of the excess hormone on the lower side of an opposing one. It is pointed out that if the suggestion that $\beta$-indoleacetic acid causes an acceleration of growth followed by an inhibitory effect, or that it may itself be converted into an inhibitor, should prove to be generally valid, it would afford a simple and satisfactory explanation of autotropism. The effects of $2: 4$-dichlorophenoxyacetic acid, ascorbic acid, $2: 3: 4$-triiodobenzoic acid and $2: 4$-dichloranisole were also investigated.

\section{STRUCTURES OF NATIVE AND MERCERIZED CELLULOSES}

\section{By DR. M. K. SEN and DR. S. C. ROY}

Indian Jute Mills Association Research Institute, Calcutta

YN a previous publication, Sen and Woods ${ }^{1}$ reported 1 the presence of two new equatorial reflexions in the diffraction pattern of native celluloses of various origins. Continuation of this work with more detailed experimentation has now made it possible to make certrin assertions with regard to the origin of one of the reflexions, $Y$. From X-ray photographs taken under various conditions of prevailing humidity, intensity and purity of radiation (including monochromatic radiation) and with different cellulose samples, such as ramie, jute, cotton, viscose and paper, it has been noted that the reflexion occurs in greater or less intensity in almost all ordinarily available cellulosic fibres, native or mercerized. Under conditions of $60-70$ per cent relative humidity and $70-75^{\circ} \mathrm{F}$. temperature, the sharpness, intensity and dispersion of the diffraction maximum are at least equal to those of certain other equatorial reflexions (Fig. 1). The observations regarding its relative intensity in different samples when compared against Meyer and Mark 101 are summarized in Table 1.
Samples
1. Defatted white jute
2. Commercially degummed ramie
3. Surgical cotton
5. Ordinary rayon
Pre-swollen rayon (treating
with ethylene diamine, wash- with ethylene diam
ing and drying)
6. Mercerized ramie
7. Mercerized jute

Table 1 Intensity
Slightly less than that of 101 reflexion. Less than that of 101. Less than that of 101 . Slightly less than that of 101 .

Greater than that of 101. Greater than that of 101 (mer-
cerized). 